Research Article

\title{
Optimized Design and Experiment on Novel Combination Vacuum and Spoon Belt Metering Device for Potato Planters
}

\author{
Zhang Wanzhi $\mathbb{D}^{1,2}$ Liu Chenglong, ${ }^{1,2}$ Lü Zhaoqin, ${ }^{1,2}$ Qi Xieteng, ${ }^{1,2}$ Lü Haoyu, ${ }^{1,2}$ \\ and Hou Jialin iD 1,2 \\ ${ }^{1}$ College of Mechanical and Electrical Engineering, Shandong Agricultural University, Tai'an 271018, China \\ ${ }^{2}$ Shandong Provincial Engineering Laboratory of Agricultural Equipment Intelligence, Tai'an 271018, China \\ Correspondence should be addressed to Hou Jialin; jlhou@sdau.edu.cn
}

Received 12 November 2019; Revised 16 February 2020; Accepted 27 February 2020; Published 23 April 2020

Academic Editor: Francesco Clementi

Copyright (c) 2020 Zhang Wanzhi et al. This is an open access article distributed under the Creative Commons Attribution License, which permits unrestricted use, distribution, and reproduction in any medium, provided the original work is properly cited.

\begin{abstract}
To improve the efficient and precise seeding of potatoes, a novel combination vacuum and spoon belt metering device was designed. The overall structure and working principle of vacuum and spoon belt metering devices were illustrated and analyzed. The stress of the potato seed situated on the vacuum and spoon belt metering device was analyzed theoretically. The structure and parameters of the key parts of the seed metering device were studied and calculated. Three-factor and three-level response surface experiments were designed based on the Box-Behnken central composite experimental design principle. After selecting seeding speed, spoon aperture, and cleaning-seed air amount as the experimental factors and selecting missing seed index, multiple seed index, and qualified seed index as the experimental indexes, the performance of the seed metering device with high efficiency and precision was verified by the experiments. The mathematical model of the response surface was established, and the influence of each factor on the performance of the seed metering device was analyzed using Design-Expert 10.0.4 software. To improve the efficient and precise seeding of potatoes, the three experimental factors were optimized. Experimental results show that the order of the factors affecting the missing seed index was seeding belt speed $>$ cleaning air pressure $>$ spoon aperture; the order of the factors affecting the multiple seed index was spoon aperture > cleaning air pressure $>$ seeding belt speed; the order of the factors affecting the qualified seed index was seeding belt speed $>$ cleaning air pressure $>$ spoon aperture; when the seeding belt speed was $0.43 \mathrm{~m} \cdot \mathrm{s}^{-1}$, the spoon aperture was $15.72 \mathrm{~mm}$, and the cleaning air pressure was $2.94 \mathrm{kPa}$, the experiment had realized potato's highly efficient and precise seeding and the missing seed index was $3.97 \%$, the multiple seed index was $4.65 \%$, and the qualified seed index was $91.38 \%$. This paper can provide a theoretical and technical reference for improving the efficient and precise seeding of potatoes.
\end{abstract}

\section{Introduction}

Potato is the world's fourth-largest food crop [1, 2], but the current level of potato planting machinery is generally not high, especially in developing countries, where the broadcast rate is only $30 \%$ [3-5]. Potato planting is a very important part of potato production $[6,7]$, and the seed meter is the core component of potato planting machinery [8]. At present, the spoon-type seed metering device is the most commonly used seeding device in the world [9]. The spoontype seed metering device has the advantages of simple operation, uniform seeding, reliability, and high work efficiency [10], but there are also shortcomings such as missing and multiple seeds. At present, almost all types of machinery require manually assisted seeding when planting potatoes, as shown in Figure 1.

To improve the performance of spoon-type seeding, domestic and foreign scholars have carried out related research studies [11-13]. In China, Lü et al. [14] designed a double-row interleaved potato seed metering for the traditional seed metering device, designed and optimized the key components, and theoretically analyzed the performance characteristics of the driving wheel and the vibration cleaning device; Wang et al. [15] improved the design of a double-row staggered spoon-type potato precision seed metering device and optimized the structural parameters of 


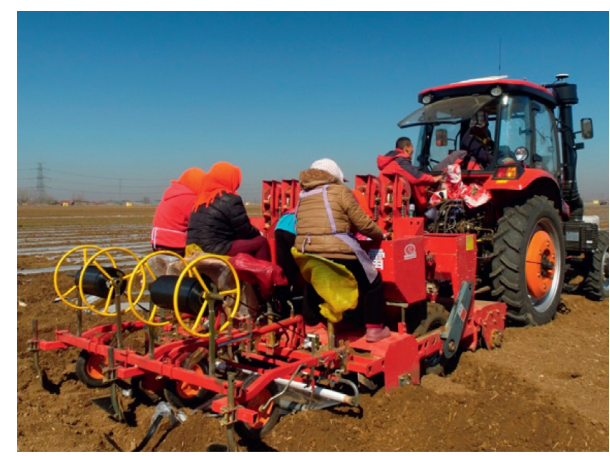

FIGURE 1: Manually assisted seeding.

the double-stitched staggered seeding assembly, active drive assembly, and vibration cleaning device; Liu et al. [16] designed a vibration-arranging based seeder for potato microseed. Based on the principle of forced vibration, potato microseeds were arranged and seeded in a single row, and the key structural and working parameters of seeding device were confirmed according to the physical characteristics of potato microseed and agronomy requirements; Lai et al. [17] designed a precision single-row air-suction planter of potato microseed. After optimizing the test parameters, when the suction negative pressure was $10 \mathrm{kPa}$, the working speed was $2.5 \mathrm{~km} / \mathrm{h}$, the qualified-seeding index was greater than $90 \%$, the miss-seeding index was less than $5 \%$, and the multipleseeding index was less than 5\%. Lü et al. [18] have designed a precision air-suction-type planter for potato and experimental results have shown that the seeding qualified index is $98 \%$, the relaying seed qualified index is $1 \%$, and the missing seed qualified index is $1 \%$, but the planter's structure is complicated and the cost is high. In the United States, Crary's scoop-chain potato planter has used hydraulic control systems and radar control systems to increase job speed and seed accuracy [19]. An air-suction potato planter has been developed by Crary of the United States, using the principle of negative pressure to suck seed potatoes for seeding [20]. In Germany, the scoop belt metering device developed by Grimme is equipped with an electronic monitoring system, which is stable in operation and can adapt to different plant spacing requirements [21]. However, the two planters have complicated structures and high costs, which are not suitable for China $[22,23]$.

The current research shows that the slow operation speed, high missing, multiple seed index, and poor adaptability are the main problems of the spoon belt metering device for potato planters [24-26]. To solve the above problems, this paper presented a combination of pneumatic and mechanical methods to study and design a novel combination vacuum and spoon belt metering device for potato planters. Besides, most of China currently uses slices potatoes for planting. Slices potatoes are likely to cause virus infection, which hinders the development of the potato industry to a certain extent, and mechanization of whole potato seeding is the future development trend. Therefore, this paper focused on the seed potatoes and included three research contents: structure and working principle, main components structural design, and bench test.

\section{Structures and Working Principle}

According to the design experience of the spoon belt metering device, the structure of a novel combination vacuum and spoon belt metering system is shown in Figure 2, which mainly includes components such as spoon, air chamber, belt, seedbox, seed cleaning system, and broken arch device. The working principle of the seed metering device is that opening the air chamber fan, the air chamber forms a negative pressure, and the surface of each spoon connected to the air chamber forms a suction force; opening the cleaning fan, the surface of seed cleaning system forms a positive pressure; the seed box is full of seed potatoes; the antiarch disc is rotated back and forth by the motor to prevent seed potato arching; the driving wheel rotates counterclockwise, and the spoons in the seeding area are moved upwards by the belt; under the action of the suction and surface support of each spoon, each seed spoon draws one or two seed potatoes in turn; when the spoon moves to the seed cleaning system, the airflow blows away the seed potato which is not affected by the suction force and falls back into the seed box under the action of the clear baffle; the seed spoon is loaded with one seed potato and falls to the seed tube under the action of the driven wheel; under the isolation of the spoon, there is only one seed potato in each independent space, and at the bottom of the seed tube, the direction of seed potato movement is changed. Based on prior design experience, the line curvature at the bottom of the seed tube is 0.25 . When the planter moves at a constant speed, the device can achieve zero-speed seeding [27]. The designed novel combination vacuum and spoon belt metering device can realize potato highly efficient and precise metering seeds and reduce missing and multiple seeds.

\section{Main Component Structural Design}

3.1. Spoon Structure Design and Arrangement. The structural parameters of the spoon mainly depend on the shape of the seed potato. In general, the maximum diameter $D$ of the spoon surface and the depth $H$ of the seed spoon should be satisfied:

$$
D>\bar{L}>\bar{T}>2 H,
$$

where $\bar{L}$ is the average length of the seed potato $(\mathrm{mm})$ and $\bar{T}$ is the average thickness of the seed potato $(\mathrm{mm})$. The length, width, and thickness of seed potatoes used in potato planting in Shandong province are generally $45 \sim 55 \mathrm{~mm}, 30 \sim 45 \mathrm{~mm}$, and $20 \sim 35 \mathrm{~mm}$. Therefore, the design of the spoon has a surface diameter of $56 \mathrm{~mm}$ and a depth of $16 \mathrm{~mm}$. The bottom of the spoon is connected to the air chamber through a circular hole. The diameter of the circular hole also directly affects the performance of the seed metering device. The diameter is too large to increase the power consumption of the fan and absorb more seed potatoes; being too small will result in insufficient adsorption. For ellipsoidal seeds, referring to the suction hole design method of the air-suctiontype seed meter on the agricultural machinery design manual [28], the diameter of the circular hole can usually be described according to the seed size: 


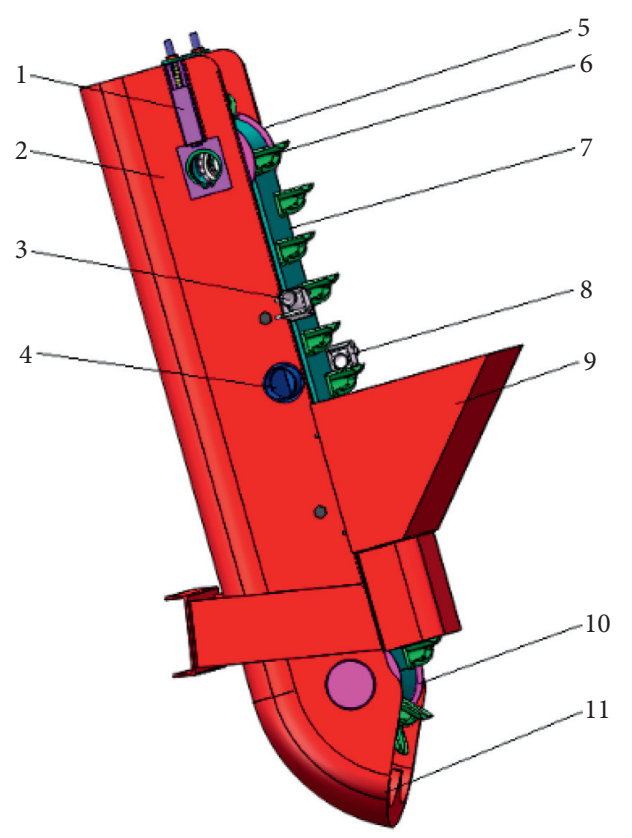

FIGURE 2: Structure of vacuum and spoon belt metering device. 1: belt tensioning device, 2: frame, 3: left cleaning tube, 4: air chamber inlet, 5: driven wheel, 6: spoon, 7: belt, 8: right cleaning rube, 9: seed box, 10: driving wheel, and 11: seeding rube.

$$
d=(0.64 \sim 0.66) \bar{T},
$$

where $d$ is the diameter of the circular hole $(\mathrm{mm})$ and $\bar{T}$ is the average thickness of the seed potato $(\mathrm{mm})$. In the paper, $d$ is selected to be three sizes of 12,15 , and $18 \mathrm{~mm}$. At the same time, to avoid the damage to the seed potato, the surface of the spoon is rounded. The structure diagram of the spoon is shown in Figure 3.

According to the potato planting process, in the ideal state, the number of seed potatoes falling into the seed furrow per unit time $\Delta t$ is equal to the seeding quantity of the seed metering device, namely,

$$
\frac{v_{0} \Delta t}{a}=\frac{v_{1} \Delta t}{b},
$$

where $v_{0}$ is the advance speed of the seeding machine $\left(\mathrm{m} \cdot \mathrm{s}^{-1}\right) ; v_{1}$ is the line speed of the seeding belt $\left(\mathrm{m} \cdot \mathrm{s}^{-1}\right) ; a$ is the theoretical grain distance $(\mathrm{mm})$; and $b$ is the spacing of the seed spoon $(\mathrm{mm})$. According to the agronomic requirements of potato planting in Shandong province, the planting grain distance is generally $200-300 \mathrm{~mm}$, the planting machine operation speed is generally $1.0-1.5 \mathrm{~m} \cdot \mathrm{s}^{-1}$, the seeding belt line speed is generally lower than $0.6 \mathrm{~m} \cdot \mathrm{s}^{-1}$, and the spacing of the spoons is generally $140 \mathrm{~mm}$. The spoons are arranged in a double row-staggered arrangement which is shown in Figure 4.

3.2. Air Chamber Structure Design and Fan Selection. The air chamber is located between the driving wheel and the driven wheel, behind the seeding belt, and its structure is shown in Figure 5. To ensure that the air chamber and the seed belt are closely fitted and, at the same time, to reduce the friction between the two, the surface of the air chamber is covered with a layer of polyethylene, and the thickness is $3 \mathrm{~mm}$. The surface of the air chamber is provided with two pores, which are connected with the round holes of the seeding spoon. One side of the chamber is connected to the fan through the air hole to generate a negative pressure when the fan is working. The XGB-2200 high-pressure vortex fan is used. The specific parameters are shown in Table 1. In the field test, the maximum fan speed is $2800 \mathrm{rpm}$, and its power can be satisfied by the rear output shaft of the tractor, thus replacing the $380 \mathrm{~V}$ power supply. The maximum negative pressure is $30 \mathrm{kPa}$, which is much higher than the negative pressure required for potato seeding $(10 \mathrm{kPa})$.

3.3. Seed Cleaning System Design and Fan Selection. The seed cleaning system adopts the principle of air blowing, which blows air at different heights on the left and right sides of the seed spoon and blows off the seed potatoes that are not sucked in the spoon. Assume that when the seed cleaning system is closed and the seed spoon passes through the air chamber at a constant speed, the seed potato is stressed as shown in Figure 6.

The seed potato heart is the origin, the vertical direction of the seed potato movement is $X$ direction, and the direction of seed potato movement is $Y$ direction. The force equation of seed potato is

$$
\begin{array}{r}
F_{n}-G \cos \gamma-F_{x}=0, \\
G \sin \gamma-F_{f}=0,
\end{array}
$$

where $G$ is the weight of seed potato $(N) ; F_{n}$ is the support force of seed spoon on seed potato $(N) ; F_{x}$ is the suction force of seed spoon on seed potato $(N) ; F_{f}$ is the friction between seed spoon and seed potato $(N)$; $\gamma$ is the inclination angle of seeding belt $\left({ }^{\circ}\right)$. When the seed cleaning system is turned on, the $z$-axis is defined perpendicular to the $X Y$ plane, and the seed potato should be subjected to the force on the $z$-axis:

$$
F_{c}-F_{f}=0,
$$

where $F_{c}$ is the blowing force of the seed cleaning system. According to $F_{f}=\mu F_{n}$, then

$$
F_{c}=\mu\left(G \cos \gamma+F_{x}\right)
$$

where $\mu$ is the coefficient of friction. Assume that the pressure difference generated on the surface of the spoon is $\Delta P$ when the fan is working; then

$$
F_{x}=\frac{\pi D^{2} \Delta P}{4} .
$$

The key to the seed cleaning system is that it cannot blow off the seed potatoes that are sucked in the spoon. Therefore, the wind system of the seed cleaning system should eventually meet

$$
F_{c} \leq \mu\left(G \cos \gamma+\frac{\pi D^{2} \Delta P}{4}\right),
$$




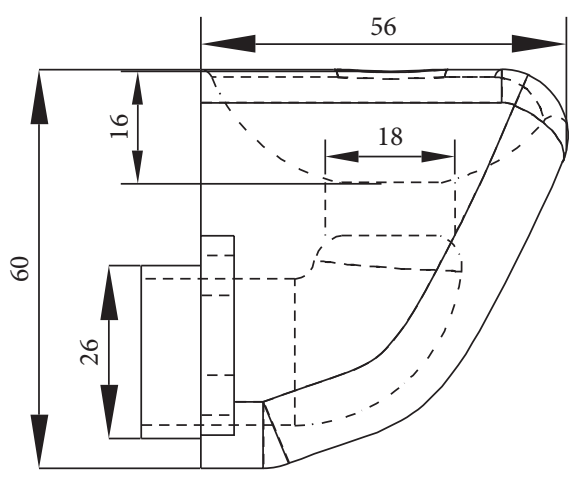

(a)

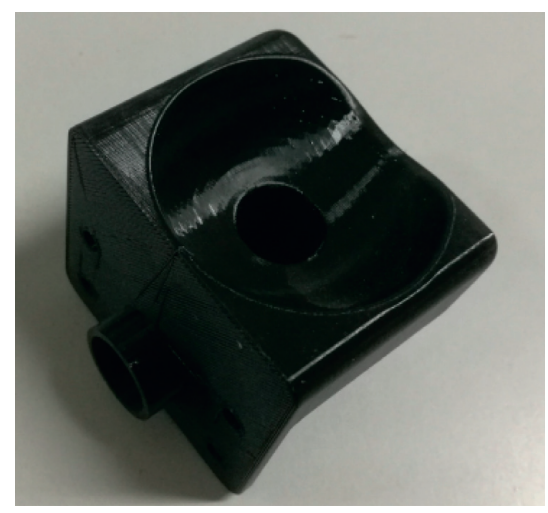

(b)

Figure 3: (a) Structure of spoon and (b) actual shape.

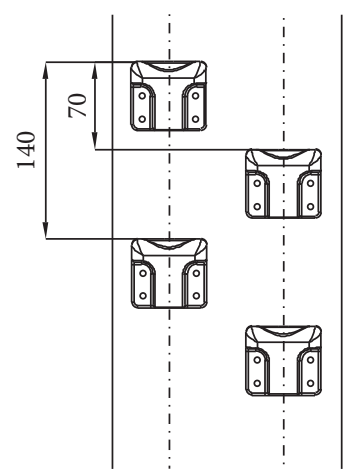

FiguRE 4: Double row-staggered arrangement of seed spoon.

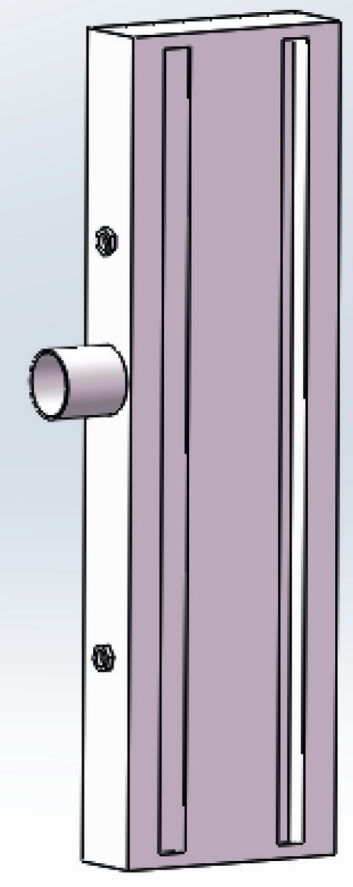

(a)

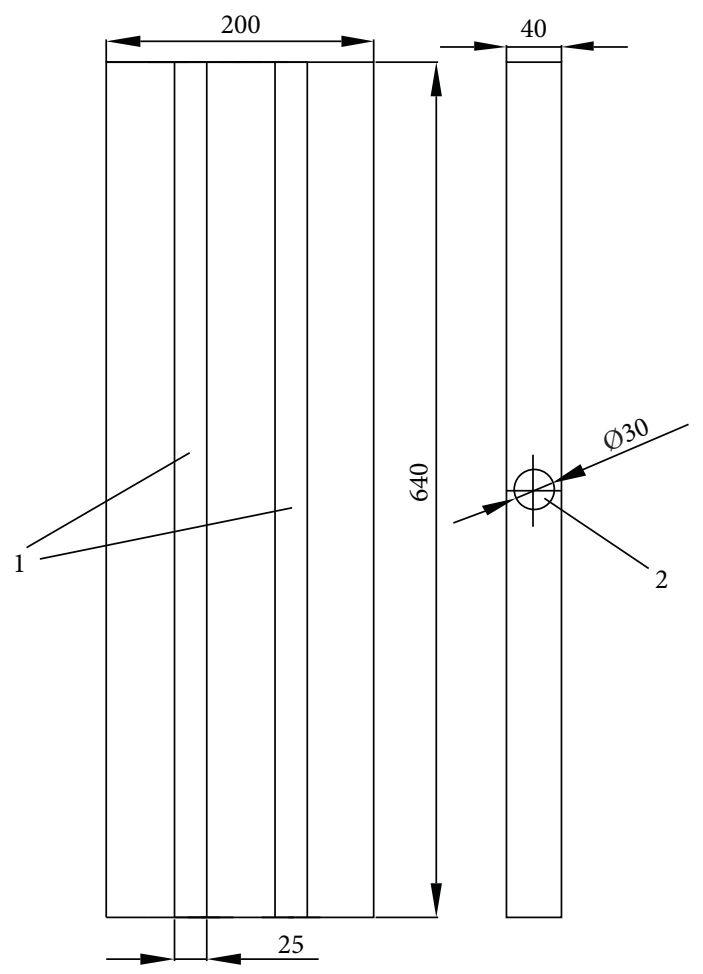

(b)

Figure 5: (a) Structure of the air chamber and (b) main dimensions of the air chamber. 1: two pores; 2: air hole. 
TABLE 1: Parameters of the XGB-2200 high-pressure vortex fan.

\begin{tabular}{lc}
\hline Parameters & Values \\
\hline Power $(\mathrm{kw})$ & 2.2 \\
Voltage $(\mathrm{v})$ & 380 \\
Maximum positive pressure $(\mathrm{kPa})$ & 36 \\
Maximum negative pressure $(\mathrm{kPa})$ & 30 \\
Air volume $\left(\mathrm{m}^{3} / \mathrm{min}\right)$ & 5 \\
Rotating speed $(\mathrm{r} / \mathrm{min})$ & 2800 \\
\hline
\end{tabular}

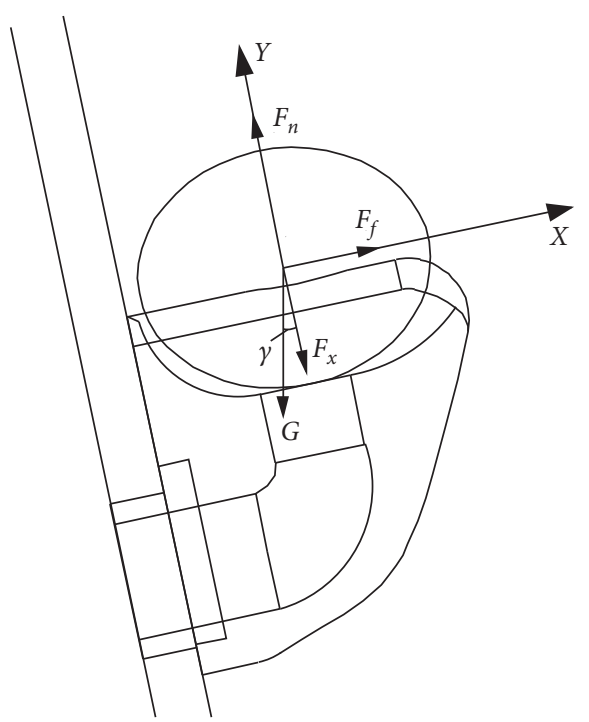

FIGURE 6: Stress analysis in the linear motion of potato.

where $\mu=0.524, G=0.035 \mathrm{~kg}, \gamma=11.5^{\circ}$, and $\Delta P=5.6 \mathrm{kpa}$. So in the experiment, the JD3901C fan has been used, the speed is $0 \sim 15000 \mathrm{r} / \mathrm{min}$, and the cleaning air pressure is $0 \sim 3.2 \mathrm{kPa}$.

3.4. Drive Assembly System Design. The drive assembly system drives the belt movement, and its structure mainly includes the driving wheel, the driven wheel, and the tensioning device. The drive assembly system adopts the driven wheel upper setting and the driving wheel lowering form. The driving wheel is connected to the three-phase asynchronous motor through the chain, and the motor can be adjusted in speed through the frequency converter. During the seeding process, the seed potato makes linear and circular motions with the belt. Therefore, the key to the design of the drive assembly system is to ensure that the seed potatoes are not thrown out during the circular motion. The force analysis of the seed potato when doing circular motion on the belt is shown in Figure 7.

The seed potato heart is the origin, the vertical direction of the seed potato movement is $X$ direction, and the direction of seed potato movement is $Y$ direction. Then the seed potato force equation is

$$
\begin{aligned}
& F_{l} \cos \alpha-F_{n}-G \sin \beta=0, \\
& F_{l} \sin \alpha-G \cos \beta-F_{f}=0,
\end{aligned}
$$

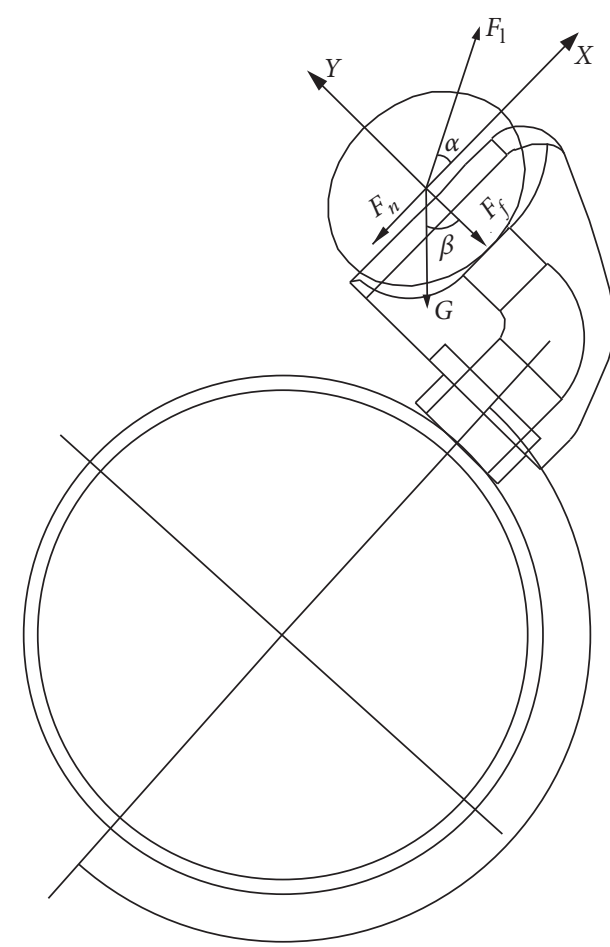

FIGURE 7: Stress analyses in the circular motion of seed potato.

where $F_{l}$ is centrifugal force; $G$ is seed potato gravity $(N) ; F_{n}$ is the seedling support force of the seed potato $(N) ; F_{f}$ is the friction between the seed spoon and the seed potato $(N) ; \alpha$ is the angle between the $F_{l}$ and $x$-axis $\left(^{\circ}\right) ; \beta$ is the angle between $G$ and $y$-axis $\left({ }^{\circ}\right)$. According to the actual movement requirements of seed potatoes, it should be satisfied in the $Y$ direction:

$$
F_{l} \sin \alpha \leq G \cos \beta+F_{f}
$$

One has

$$
\begin{aligned}
F_{l} & =\frac{m v^{2}}{R \times 10^{-3}}, \\
F_{f} & =\mu F_{n} .
\end{aligned}
$$

The above formula can be obtained

$$
R \geq \frac{v^{2}(\sin \alpha+\mu \cos \alpha)}{g(\cos \beta+\mu \sin \beta)} \times 10^{3},
$$

where $\mu=0.524, g=9.8 \mathrm{~m} \cdot \mathrm{s}^{-2}, v$ maximum value is taken as $0.5, \alpha=30^{\circ}, \beta=90^{\circ}$, and $R \geq 66$. According to the spacing of the spoon and the line speed of the belt, the radius of the driven wheel is determined to be $70 \mathrm{~mm}$.

3.5. Broken Arch System Design. To prevent seed potatoes bridging in the seedbox, a stepping motor is used to drive the disk to rotate left and right to improve the fluidity of the seed potatoes. The depth of the seedbox is $480 \mathrm{~mm}$, so the diameter of the disc is $160 \mathrm{~mm}$, and there are two levers under the disc. According to the size of the seed potatoes, the distance between the levers is $90 \mathrm{~mm}$, the length of the 
levers is $150 \mathrm{~mm}$, and the swing angle is $30^{\circ}$. The diameter of the levers affects the fluidity of the seed potatoes and the best value is $15 \mathrm{~mm}$ according to the experimental result. The stepper motor model is an 86BYG250D stepping motor. The schematic of the broken arch system is shown in Figure 8.

\section{Bench Test}

4.1. Test Materials and Methods. The Netherlands 15 has been selected as the test variety, and 600 seed potatoes with 30-40 g per seed have been selected using an electronic balance for testing. The main dimensions of the seeds are shown in Table 2 . The seeding test bench includes a vacuum and spoon belt metering device, a cleaning fan, a driving motor of the cleaning fan, a high-pressure vortex fan, and a driving motor of high-pressure vortex fan, as shown in Figure 9.

According to the Box-Behnken test principle [29], threefactor and three-level quadratic regression orthogonal test schemes have been designed. In the test, the speed of the seeding belt $x_{1}$, the aperture of the seeding spoon $x_{2}$, and the amount of fresh air volume $x_{3}$ were selected as the influencing factors of the test. According to the current potato planter working speed generally does not exceed $1.5 \mathrm{~m} \cdot \mathrm{s}^{-1}$, the plant spacing is $200 \mathrm{~mm}$, and the test selects the belt speed $0.3 \sim 0.5 \mathrm{~m} \cdot \mathrm{s}^{-1}$, the spoon aperture $12 \sim 18 \mathrm{~mm}$, and the cleaning air pressure 1.6 3.2 kPa. Test levels and factors are shown in Table 3.

The missing seed index $Y_{1}$, the multiple seed index $Y_{2}$, and the qualified seed index $Y_{3}$ are selected as each test evaluation indicator, and the calculation formula is

$$
\begin{aligned}
& Y_{1}=\frac{n_{1}}{N} \times 100 \%, \\
& Y_{2}=\frac{n_{2}}{N} \times 100 \%, \\
& Y_{3}=\frac{n_{3}}{N} \times 100 \%,
\end{aligned}
$$

where $n_{1}$ is the number of single seed potato; $n_{2}$ is the number of nonseed potatoes; $n_{3}$ is the number of multiple seed potatoes; $N$ is the number of seed potatoes; here it is 600.

\subsection{Test Results and Analysis}

4.2.1. Test Results. The three-factor and three-level test protocol included 17 trials (5 zero-point estimation errors; 12 analysis factors), and the test protocol and results are shown in Table 4.

4.2.2. Regression Model Establishment and Optimization. According to the test data shown in Table 5, multiple regression analysis has been performed on the test data under Design-Expert 10.0.4 software to establish a quadratic polynomial response surface regression model, as follows:

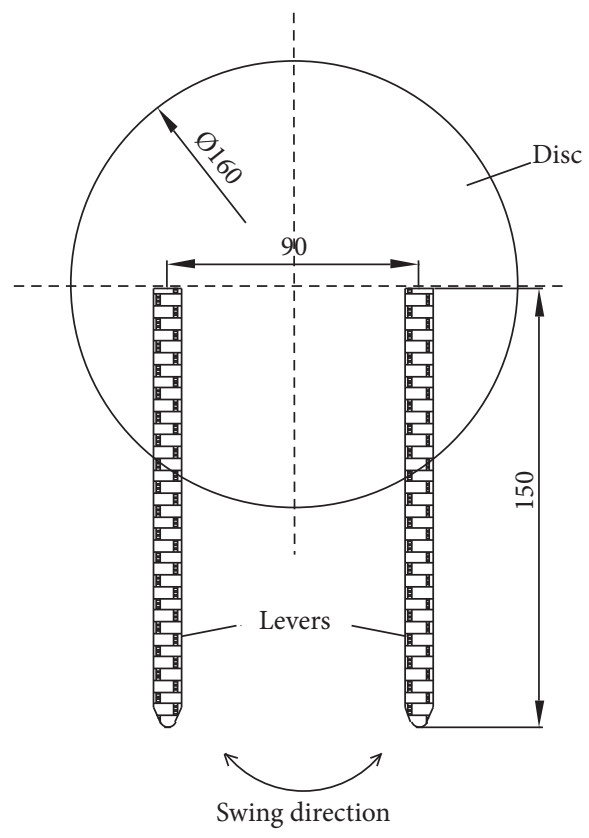

FIGURE 8: The schematic of the broken arch system.

$$
\begin{aligned}
Y_{1}= & 2.74+1.33 x_{1}-0.49 x_{2}+0.66 x_{3}-0.1 x_{1} x_{2}-0.6 x_{1} x_{3} \\
& +0.53 x_{2} x_{3}-0.36 x_{1}^{2}+0.42 x_{2}^{2}+1.17 x_{3}^{2}, \\
Y_{2}= & 5.32+0.96 x_{1}+1.15 x_{2}-1.36 x_{3}-1.75 x_{1} x_{2} \\
& \quad-0.43 x_{1} x_{3}+0.1 x_{2} x_{3}+1.43 x_{1}^{2}+1.0 x_{2}^{2}-0.37 x_{3}^{2} \\
Y_{3}= & 91.94-2.29 x_{1}-0.66 x_{2}+0.7 x_{3}+1.85 x_{1} x_{2} \\
& +1.02 x_{1} x_{3}-0.63 x_{2} x_{3}-1.07 x_{1}^{2}-1.42 x_{2}^{2}-0.8 x_{3}^{2} .
\end{aligned}
$$

The regression equation has been analyzed by variance, as shown in Table 5.

According to Table 5, the significant level $P$ values of the missing seed index $Y_{1}$, the multiple seed index $Y_{2}$, and the qualified seed index $Y_{3}$ are all less than 0.01 , indicating that the regression model established in the paper is highly significant; lack of fit in the significant level is all greater than 0.05 , indicating that the regression equation is highly fitted; the decision coefficients $R^{2}$ are $0.9550,0.9475$, and 0.9709 , respectively, indicating that the three models built in the paper can explain more than $94 \%$ of the evaluation indicators. Therefore, according to the significance level of each factor in Table 5, the factors of $P<0.05$ are selected for model optimization, and finally

$$
\begin{aligned}
Y_{1}= & 2.74+1.33 x_{1}-0.49 x_{2}+0.66 x_{3}-0.6 x_{1} x_{3} \\
& +0.53 x_{2} x_{3}+1.17 x_{3}^{2}, \\
Y_{2}= & 5.32+0.96 x_{1}+1.15 x_{2}-1.36 x_{3}-1.75 x_{1} x_{2} \\
& +1.43 x_{1}^{2}+1.0 x_{2}^{2},
\end{aligned}
$$


TABle 2: Main dimensions of the seeds.

\begin{tabular}{lccc}
\hline Direction & Maximum value $(\mathrm{mm})$ & Minimum value $(\mathrm{mm})$ & Mean value $(\mathrm{mm})$ \\
\hline Length $\mathrm{L}$ & 55.07 & 45.28 & 49.71 \\
Width $K$ & 44.06 & 32.64 & 37.38 \\
Thickness $T$ & 29.65 & 22.65 & 25.95 \\
\hline
\end{tabular}

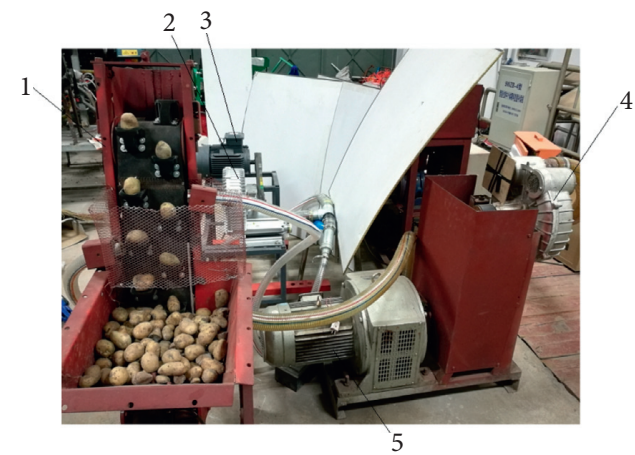

FIGURE 9: Seed metering test bench, 1: vacuum and spoon belt metering device, 2: cleaning fan, 3: driving motor of cleaning fan, 4: highpressure vortex fan, and 5: driving motor of high-pressure vortex fan.

TABle 3: Test levels and factors.

\begin{tabular}{lccc}
\hline Test levels & Belt speed $x_{1}\left(\mathrm{~m} \cdot \mathrm{s}^{-1}\right)$ & Spoon aperture $x_{2}(\mathrm{~mm})$ & Cleaning air pressure $x_{3}(\mathrm{kPa})$ \\
\hline-1 & 0.3 & 12 & 1.6 \\
0 & 0.4 & 15 & 2.4 \\
1 & 0.5 & 18 & 3.2 \\
\hline
\end{tabular}

TABLE 4: Test protocol and response values.

\begin{tabular}{|c|c|c|c|c|c|c|}
\hline \multirow[b]{2}{*}{$\begin{array}{l}\text { Test } \\
\text { number }\end{array}$} & \multicolumn{3}{|c|}{ Factor level } & \multicolumn{3}{|c|}{ Response values } \\
\hline & $\begin{array}{c}\text { Belt speed } \\
x_{1} \\
\end{array}$ & $\begin{array}{c}\text { Spoon aperture } \\
x_{2} \\
\end{array}$ & $\begin{array}{c}\text { Cleaning air pressure } \\
x_{3}\end{array}$ & $\begin{array}{l}\text { Missing seed index } \\
(\%)\end{array}$ & $\begin{array}{l}\text { Multiple seed index } \\
(\%)\end{array}$ & $\begin{array}{l}\text { Qualified seed index } \\
(\%)\end{array}$ \\
\hline 1 & 0 & 0 & 0 & 2.5 & 5.6 & 91.9 \\
\hline 2 & -1 & 1 & 0 & 1.2 & 9.4 & 89.4 \\
\hline 3 & 0 & 0 & 0 & 2.5 & 4.5 & 93.0 \\
\hline 4 & 1 & 1 & 0 & 3.6 & 8.2 & 88.2 \\
\hline 5 & 0 & 0 & 0 & 2.8 & 5.7 & 91.5 \\
\hline 6 & -1 & -1 & 0 & 1.8 & 3.8 & 94.4 \\
\hline 7 & 0 & -1 & 1 & 5.4 & 2.8 & 91.8 \\
\hline 8 & 0 & -1 & -1 & 4.4 & 6.6 & 89.0 \\
\hline 9 & 0 & 0 & 0 & 3.1 & 5.9 & 91.0 \\
\hline 10 & -1 & 0 & -1 & 1.3 & 6.1 & 92.6 \\
\hline 11 & 0 & 1 & -1 & 2.2 & 8.9 & 88.9 \\
\hline 12 & 1 & 0 & 1 & 4.6 & 5.8 & 89.6 \\
\hline 13 & 0 & 0 & 0 & 2.8 & 4.9 & 92.3 \\
\hline 14 & 0 & 1 & 1 & 5.3 & 5.5 & 89.2 \\
\hline 15 & 1 & -1 & 0 & 4.6 & 9.6 & 85.8 \\
\hline 16 & -1 & 0 & 1 & 3.1 & 5.1 & 91.8 \\
\hline 17 & 1 & 0 & -1 & 5.2 & 8.5 & 86.3 \\
\hline
\end{tabular}

$$
\begin{aligned}
Y_{3}= & 91.94-2.29 x_{1}-0.66 x_{2}+0.7 x_{3}+1.85 x_{1} x_{2} \\
& +1.02 x_{1} x_{3}-1.07 x_{1}^{2}-1.42 x_{2}^{2}-0.8 x_{3}^{2} .
\end{aligned}
$$

According to formulas (22)-(24), it can be seen that, in addition to seeding belt speed $x_{1}$, aperture of the seeding spoon $x_{2}$, and cleaning air pressure $x_{3}$, the significant factors affecting the missing seed index $Y_{1}$ include the interaction terms of seeding belt speed and aperture of the seeding spoon $\left(x_{1} x_{2}\right)$, aperture of the seeding spoon and cleaning air pressure $\left(x_{2} x_{3}\right)$, and the quadratic term of cleaning air pressure $x_{3}$; the significant factors affecting the multiple seed 
TABLE 5: Variance analysis of regression equation.

\begin{tabular}{|c|c|c|c|c|c|c|c|c|c|c|c|c|}
\hline \multirow[b]{2}{*}{ Source } & \multicolumn{4}{|c|}{ Missing seed index $Y_{1}$} & \multicolumn{4}{|c|}{ Multiple seed index $Y_{2}$} & \multicolumn{4}{|c|}{ Qualified seed index $Y_{3}$} \\
\hline & $\begin{array}{l}\text { Sum of } \\
\text { squares }\end{array}$ & $\begin{array}{l}\text { Degree of } \\
\text { freedom }\end{array}$ & $\begin{array}{c}F \\
\text { value }\end{array}$ & $\begin{array}{l}\text { Significant } \\
\text { level } P\end{array}$ & $\begin{array}{l}\text { Sum of } \\
\text { squares }\end{array}$ & $\begin{array}{c}\text { Degree of } \\
\text { freedom }\end{array}$ & $\begin{array}{c}F \\
\text { value }\end{array}$ & $\begin{array}{l}\text { Significant } \\
\text { level } P\end{array}$ & $\begin{array}{l}\text { Sum of } \\
\text { squares }\end{array}$ & $\begin{array}{l}\text { Degree of } \\
\text { freedom }\end{array}$ & $\begin{array}{c}F \\
\text { value }\end{array}$ & $\begin{array}{c}\text { Significant } \\
\text { level } P\end{array}$ \\
\hline Model & 29.06 & 9 & 16.50 & $0.0006^{* *}$ & 59.58 & 9 & 14.04 & $0.0011^{* *}$ & 86.46 & 9 & 25.97 & $0.0001^{* *}$ \\
\hline$x_{1}$ & 14.05 & 1 & 71.79 & $<0.0001^{* *}$ & 7.41 & 1 & 15.72 & $0.0054^{* *}$ & 41.86 & 1 & 113.16 & $<0.0001^{* *}$ \\
\hline$x_{2}$ & 1.90 & 1 & 9.72 & $0.0169^{*}$ & 10.58 & 1 & 22.44 & $0.0021^{* *}$ & 3.51 & 1 & 9.49 & $0.0178^{*}$ \\
\hline$x_{3}$ & 3.51 & 1 & 17.95 & $0.0039^{* *}$ & 14.85 & 1 & 31.50 & $0.0008^{* *}$ & 3.92 & 1 & 10.60 & $0.0140^{*}$ \\
\hline$x_{1} x_{2}$ & 0.040 & 1 & 0.20 & 0.6648 & 12.25 & 1 & 25.98 & $0.0014^{* *}$ & 13.69 & 1 & 37.01 & $0.0005^{* *}$ \\
\hline$x_{1} x_{3}$ & 1.44 & 1 & 7.36 & $0.0301^{*}$ & 0.72 & 1 & 1.53 & 0.2557 & 4.20 & 1 & 11.36 & $0.0119^{*}$ \\
\hline$x_{2} x_{3}$ & 1.10 & 1 & 5.64 & $0.0493^{*}$ & 0.040 & 1 & 0.085 & 0.7793 & 1.56 & 1 & 4.22 & 0.0789 \\
\hline$x_{1}^{2}$ & 0.54 & 1 & 2.75 & 0.1412 & 8.58 & 1 & 18.20 & $0.0037^{* *}$ & 4.82 & 1 & 13.03 & $0.0086^{* *}$ \\
\hline$x_{2}^{\frac{1}{2}}$ & 0.73 & 1 & 3.75 & 0.0940 & 4.23 & 1 & 8.97 & $0.0201^{*}$ & 8.49 & 1 & 22.95 & $0.0020^{* *}$ \\
\hline$x_{3}^{2}$ & 5.74 & 1 & 29.34 & $0.0010^{* *}$ & 0.58 & 1 & 1.24 & 0.3024 & 2.66 & 1 & 7.19 & $0.0314^{*}$ \\
\hline Residual & 1.37 & 7 & & & 3.30 & 7 & & & 2.59 & 7 & & \\
\hline $\begin{array}{l}\text { Lack of } \\
\text { fit }\end{array}$ & 1.12 & 3 & 5.91 & 0.0594 & 1.89 & 3 & 1.79 & 0.2880 & 0.26 & 3 & 0.15 & 0.9263 \\
\hline $\begin{array}{l}\text { Pure } \\
\text { error }\end{array}$ & 0.25 & 4 & & & 1.41 & 4 & & & 2.33 & 4 & & \\
\hline Total & 30.42 & 16 & & & 62.88 & 16 & & & 89.05 & 16 & & \\
\hline
\end{tabular}

Note. $P<0.01$ (highly significant, ${ }^{* *}$ ); $P<0.05$ (significant, ${ }^{*}$ ).

index $Y_{2}$ include the interaction terms of seeding belt speed and aperture of the seeding spoon $\left(x_{1} x_{2}\right)$, the quadratic term of seeding belt speed $x_{1}$, and the aperture of the seeding spoon $x_{2}$; the significant factors affecting the qualified seed index $Y_{3}$ include the interaction terms of seeding belt speed and aperture of the seeding spoon $\left(x_{1} x_{2}\right)$, seeding belt speed and cleaning air pressure $\left(x_{1} x_{3}\right)$, and the quadratic term of seeding belt speed $x_{1}$, the aperture of the seeding spoon $x_{2}$, and cleaning air pressure $x_{3}$.

4.2.3. Interaction Factor Impact Analysis. According to the results of the regression equation analysis, the response surface map was drawn under Design-Expert 10.0.4 software, and the influence of the seeding belt speed $x_{1}$, the aperture of the seeding spoon $x_{2}$, and the cleaning air pressure $x_{3}$ on the response value were analyzed.

The response surface curves of the effect of the seeding belt speed $x_{1}$, the aperture of the seeding spoon $x_{2}$, and the cleaning air pressure $x_{3}$ on the missing seed index $Y_{1}$ are shown in Figures 10(a)-10(c). Figure 10(a) shows the effect of the seeding belt speed $x_{1}$ and the aperture of the seeding spoon $x_{2}$ on the missing seed index $Y_{1}$ when the cleaning air pressure $x_{3}$ is equal to $2.4 \mathrm{kPa}$. It can be seen that increasing the aperture of the seeding spoon $x_{2}$ and reducing the speed of the seeding belt $x_{1}$ can reduce the missing seed index $Y_{1}$. Figure 10(b) shows the effect of the seeding belt speed $x_{1}$ and the cleaning air pressure $x_{3}$ on the missing seed index $Y_{1}$ when the aperture of the seeding spoon $x_{2}$ is equal to $15 \mathrm{~mm}$. It can be seen that the missing seed index $Y_{1}$ increases with the increase of the cleaning air pressure $x_{3}$ and the seeding belt speed $x_{1}$. Figure 10(c) shows the effect of the aperture of the seeding spoon $x_{2}$ and the cleaning air pressure $x_{3}$ on the missing seed index $Y_{1}$ when the seeding belt speed $x_{1}$ is equal to $0.4 \mathrm{~m} \cdot \mathrm{s}^{-1}$. It can be seen that increasing the aperture of the seeding spoon $x_{2}$ and reducing the cleaning air pressure $x_{3}$ can reduce the missing seed index $Y_{1}$. It can be further seen in Table 5 that reducing the speed of the seed belt $x_{1}$, increasing the aperture of the seeding spoon $x_{2}$, and the cleaning air pressure $x_{3}$ can help reduce the missing seed index $Y_{1}$. The contribution rate of the three factors is the seeding belt speed $x_{1}>$ cleaning air pressure $x_{3}>$ aperture of the seeding spoon $x_{2}$.

The response surface curves of the effect of the seeding belt speed $x_{1}$, the aperture of the seeding spoon $x_{2}$, and the cleaning air pressure $x_{3}$ on the multiple seed index $Y_{2}$ are shown in Figures 11(a)-11(c). Figure 11(a) shows the effect of the seeding belt speed $x_{1}$ and the aperture of the seeding spoon $x_{2}$ on the multiple seed index $Y_{2}$ when the cleaning air pressure $x_{3}$ is equal to $2.4 \mathrm{kPa}$. It can be seen that reducing the aperture of the seeding spoon $x_{2}$ and increasing the speed of the seeding belt $x_{1}$ can help reduce multiple seed index $Y_{2}$. Figure 11(b) shows the effect of the seeding belt speed $x_{1}$ and the cleaning air pressure $x_{3}$ on the multiple seed index $Y_{2}$ when the aperture of the seeding spoon $x_{2}$ is equal to $15 \mathrm{~mm}$. It can be seen that increasing the cleaning air pressure $x_{3}$ and the seeding belt speed $x_{1}$ can also reduce the multiple seed index $Y_{2}$; Figure 11(c) shows the effect of the aperture of the seeding spoon $x_{2}$ and the cleaning air pressure $x_{3}$ on the multiple seed index $Y_{2}$ when the seeding belt speed $x_{1}$ is equal to $0.4 \mathrm{~m} \cdot \mathrm{s}^{-1}$. It can be seen that the smaller the cleaning air pressure $x_{3}$ and the larger the aperture of the seeding spoon $x_{2}$, the higher the multiple seed index $Y_{2}$. Combined with Table 5, it can be seen that increasing the speed of seeding belt $x_{1}$, reducing the aperture of the seeding spoon $x_{2}$, and increasing the cleaning air pressure $x_{3}$ can help reduce the multiple seed index $Y_{2}$. The contribution rate of the three factors is aperture of the seeding spoon $x_{2}>$ cleaning air pressure $x_{3}>$ speed of seeding belt $x_{1}$.

The response surface curves of the effect of the seeding belt speed $x_{1}$, the aperture of the seeding spoon $x_{2}$, and the cleaning air pressure $x_{3}$ on the qualified seed index $Y_{3}$ are shown in Figures 12(a)-12(c). It can be seen that the qualified seed index $Y_{3}$ increases first and then decreases slightly with 


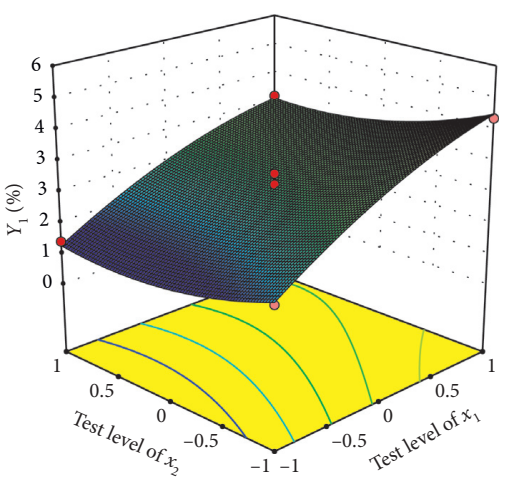

(a)

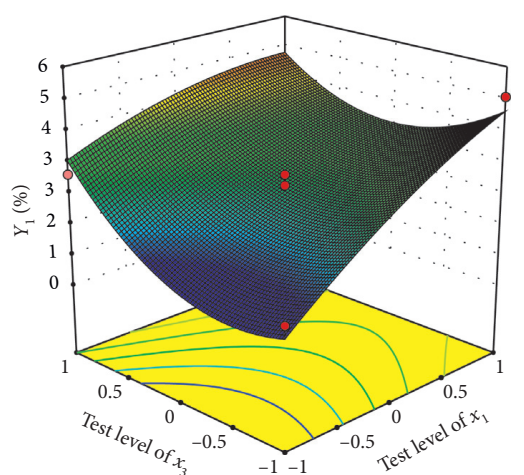

(b)

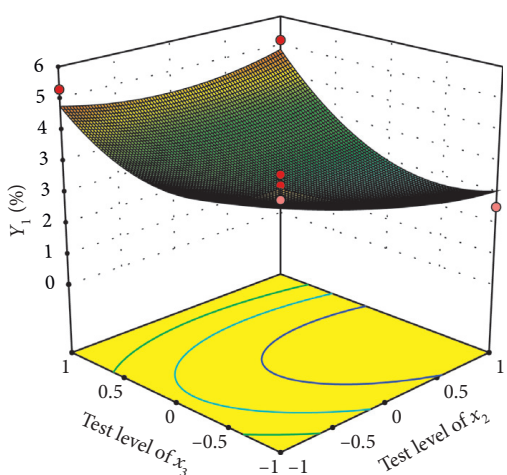

(c)

FIgURE 10: (a) The effect of the seeding belt speed $x_{1}$ and the aperture of the seeding spoon $x_{2}$ on the missing seed index $Y_{1}$ when the cleaning air pressure $x_{3}$ is equal to $2.4 \mathrm{kPa}$, (b) the effect of the seeding belt speed $x_{1}$ and the cleaning air pressure $x_{3}$ on the missing seed index $Y_{1}$ when the aperture of the seeding spoon $x_{2}$ is equal to $15 \mathrm{~mm}$, and (c) the effect of the aperture of the seeding spoon $x_{2}$ and the cleaning air pressure $x_{3}$ on the missing seed index $Y_{1}$ when the seeding belt speed $x_{1}$ is equal to $0.4 \mathrm{~m} \cdot \mathrm{s}^{-1}$.

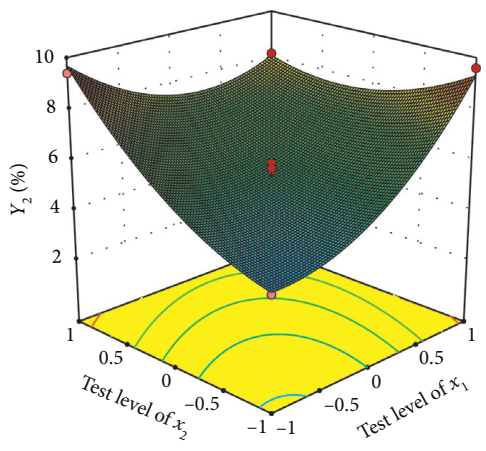

(a)

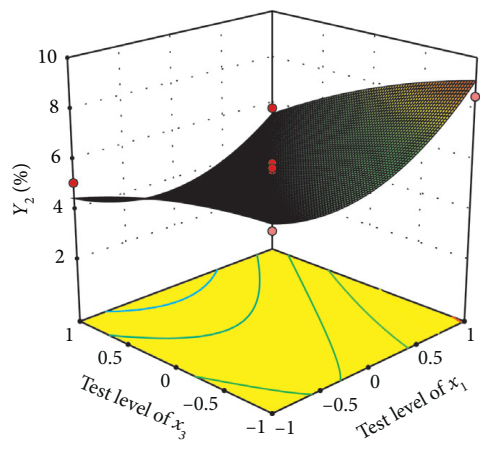

(b)

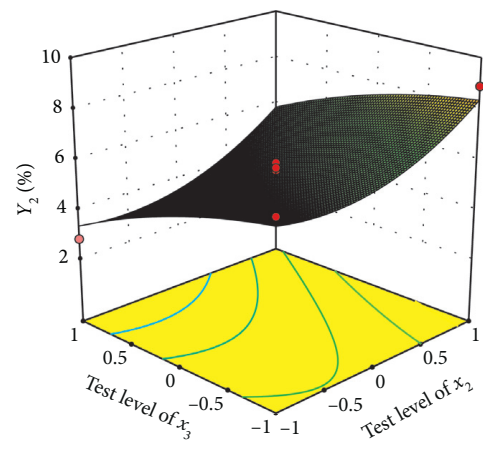

(c)

FIgURE 11: (a) The effect of the seeding belt speed $x_{1}$ and the aperture of the seeding spoon $x_{2}$ on the multiple seed index $Y_{2}$ when the cleaning air pressure $x_{3}$ is equal to $2.4 \mathrm{kPa}$, (b) the effect of the seeding belt speed $x_{1}$ and the cleaning air pressure $x_{3}$ on the multiple seed index $Y_{2}$ when the aperture of the seeding spoon $x_{2}$ is equal to $15 \mathrm{~mm}$, and (c) the effect of the aperture of the seeding spoon $x_{2}$ and the cleaning air pressure $x_{3}$ on the multiple seed index $Y_{2}$ when the seeding belt speed $x_{1}$ is equal to $0.4 \mathrm{~m} \cdot \mathrm{s}^{-1}$.

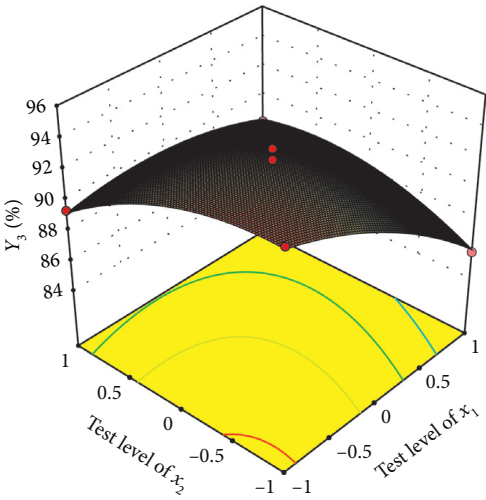

(a)

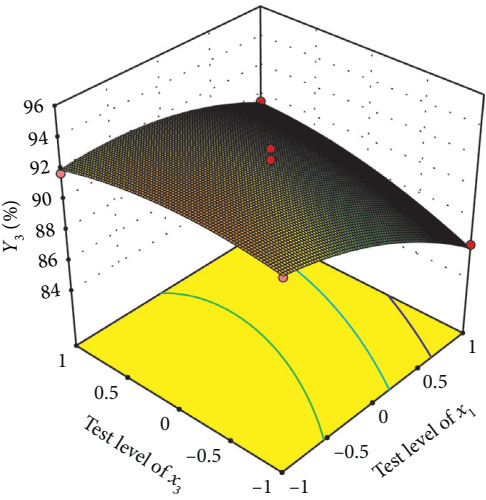

(b)

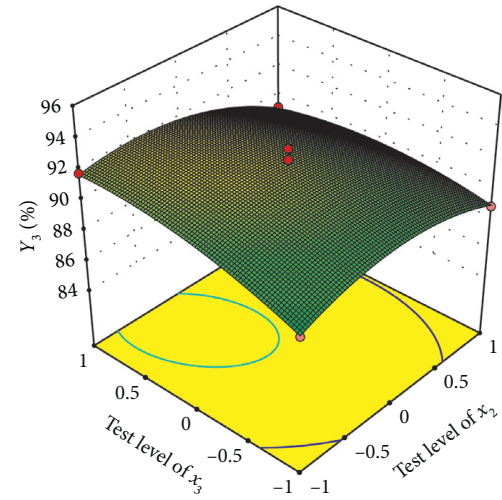

(c)

FIgURE 12: (a) The effect of the seeding belt speed $x_{1}$ and the aperture of the seeding spoon $x_{2}$ on the qualified seed index $Y_{3}$ when the cleaning air pressure $x_{3}$ is equal to $2.4 \mathrm{kPa}$, (b) the effect of the seeding belt speed $x_{1}$ and the cleaning air pressure $x_{3}$ on the qualified seed index $Y_{3}$ when the aperture of the seeding spoon $x_{2}$ is equal to $15 \mathrm{~mm}$, and (c) the effect of the aperture of the seeding spoon $x_{2}$ and the cleaning air pressure $x_{3}$ on the qualified seed index $Y_{3}$ when the seeding belt speed $x_{1}$ is equal to $0.4 \mathrm{~m} \cdot \mathrm{s}^{-1}$. 
TABLe 6: Optimization of test results.

\begin{tabular}{lccc}
\hline Project & Missing seed index (\%) & Multiple seed index (\%) & Qualified seed index (\%) \\
\hline Test 1 & 3.59 & 4.72 & 91.69 \\
Test 2 & 3.98 & 5.28 & 90.74 \\
Test 3 & 4.05 & 4.42 & 91.53 \\
Test 4 & 4.15 & 4.91 & 90.94 \\
Test 5 & 3.58 & 4.86 & 91.56 \\
Average value & 3.91 & 4.84 & 91.29 \\
Optimization value & 3.97 & 4.65 & 91.38 \\
Relative error & 1.51 & 4.09 & 0.09 \\
\hline
\end{tabular}

the decrease of the three factors. It can be further seen in Table 5 that the contribution rate of the three factors is seeding belt speed $x_{1}>$ cleaning air pressure $x_{3}>$ aperture of the seeding spoon $x_{2}$, and the seeding belt speed $x_{1}$ is the main factor affecting the qualified seed index $Y_{3}$.

4.3. Model Optimization and Test Verification. According to GB/T 6242-2006 "testing methods for potato planters" and Y/T1415-2007 "technical specifications for potato planter quality evaluation", to meet the requirements of precise and high-efficiency seeding, the planting space is set to $200 \mathrm{~mm}$, the planting machine speed is at least $1.2 \mathrm{~m} \cdot \mathrm{s}^{-1}$, and the seeding belt speed should be greater than $0.42 \mathrm{~m} \cdot \mathrm{s}^{-1}$. To meet the requirements, the paper further seeks the best combination of parameters to achieve the lowest missing seed index and multiple seed index and the highest qualified seed index. Combined with the boundary conditions of the test factors, the optimization constraints are defined as

$$
\begin{cases}\min Y_{1} & \\ \min Y_{2} & \\ \max Y_{3} & \\ \text { s.t. } & 0.42 \mathrm{~m} \cdot \mathrm{s}^{-1} \leq x_{1} \leq 0.5 \mathrm{~m} \cdot \mathrm{s}^{-1} \\ & 12.0 \mathrm{~mm} \leq x_{2} \leq 18.0 \mathrm{~mm} \\ & 1.6 \mathrm{~m}^{3} \cdot \mathrm{min}^{-1} \leq x_{3} \leq 2.8 \mathrm{~m}^{3} \cdot \mathrm{min}^{-1} \\ & 0<Y_{i}<1, \quad i=1,2,3\end{cases}
$$

The optimization of each parameter is performed by using Design-Expert 10.0.4 software. Based on the BoxBehnken response surface analysis, the optimization process mainly uses the quadratic polynomial fitting method to obtain the fitting equation, and the equation is solved to obtain the optimal value. When the seeding belt speed is $0.43 \mathrm{~m} \cdot \mathrm{s}^{-1}$, the aperture of the seeding spoon is $15.72 \mathrm{~mm}$, and the cleaning air pressure is $2.94 \mathrm{kPa}$, the high-efficiency precision seeding requirement is met. At this time, the missing seed index is $3.97 \%$, the multiple seed index is $4.65 \%$, and the qualified seed index is $91.38 \%$. To verify the accuracy of the optimization scheme, set the seeding belt speed to $0.43 \mathrm{~m} \cdot \mathrm{s}^{-1}$, the aperture of the seeding spoon to $15.72 \mathrm{~mm}$, and the cleaning air pressure to $2.94 \mathrm{kPa}$ for 5 repeated tests. The test results are shown in Table 6 . The test values of the respective response values are in good agreement with the optimized values, and the relative errors are less than $5 \%$. When the seeding belt speed is $0.43 \mathrm{~m} \cdot \mathrm{s}^{-1}$, the aperture of the seeding spoon is $15.72 \mathrm{~mm}$, and the cleaning air pressure is $2.64 \mathrm{kPa}$, the average missing seed index is $3.91 \%$, the average multiple seed index is $4.84 \%$, and the average qualified seed index is $91.29 \%$, which is better than the agricultural industry standard.

The effect of the designed seeding device also is compared with the existing seed metering device. Compared with the current seeding rate of $1.67 \mathrm{~m} \cdot \mathrm{s}^{-1}$ and the qualified seed index of $89.92 \%$, the seeding rate of the seeding device designed in this paper is $1.79 \mathrm{~m} \cdot \mathrm{s}^{-1}$ and the qualified seed index is $91.29 \%$. The speed is increased by $7.2 \%$ and the qualified seed index is increased by $1.5 \%$.

\section{Conclusions}

(1) This paper designed a kind of aspiration-spoon-belt device for potato high-efficiency precision seed metering. The overall structure and working principle of the aspiration-spoon-belt device were illustrated and analyzed. The stress of the potato seed situated on the aspiration-spoon-belt was analyzed theoretically. The structure and parameters of the key parts of the seed metering device were studied and calculated.

(2) Three-factor and three-level response surface experiments were designed based on the Box-Behnken central composite experimental design principle. After selecting seeding speed, spoon aperture, and cleaning-seed air amount as the experimental factors and selecting missing seed index, multiple seed index, and qualified seed index as the experimental indexes, the performance of the seed metering device with high efficiency and precision was verified by the experiments.

(3) A response surface mathematical model was built using Design-Expert 10.0.4 software. The response surface model $P$ values in the response surface model were less than 0.01 , indicating that the regression model established in the paper was highly significant; the missing items were all greater than 0.05 , indicating that the regression equation was highly fitted; the decision coefficients $R 2$ were $0.9550,0.9475$, and 0.9709 , respectively, indicating that the three models built in the paper could explain more than $94 \%$ of the evaluation indicators.

(4) Interaction factor impact analysis showed that the order of the factors affecting the missing seed index 
was seeding belt speed $>$ cleaning air pressure $>$ spoon aperture; the order of the factors affecting the multiple seed index was spoon aperture $>$ cleaning air pressure $>$ seeding belt speed; the order of the factors affecting the qualified seed index was seeding belt speed $>$ cleaning air pressure $>$ spoon aperture; the seeding belt speed is the main factor affecting the qualified seed index.

(5) When the seeding belt speed was $0.43 \mathrm{~m} \cdot \mathrm{s}^{-1}$, the aperture of the seeding spoon was $15.72 \mathrm{~mm}$, and the cleaning air pressure was $2.64 \mathrm{kPa}$, the high-efficiency precision seeding requirement was met. At this time, the missing seed index was $3.97 \%$, the multiple seed index was $4.65 \%$, and the qualified seed index was $91.38 \%$, which was better than the agricultural industry standard.

\section{Data Availability}

The digital data supporting this article are from previously reported studies and datasets, which have been cited.

\section{Conflicts of Interest}

The authors declare that they have no conflicts of interest.

\section{Acknowledgments}

This work was supported by the Intelligent Agricultural Machinery Equipment Special Project "Potato Precision Seeding Technology and Equipment Research and Development" of the 13th Five-Year National Key Research Development Project (2017YFD0700705), Shandong Provincial Natural Science Foundation (ZR2019BC018), Key Research and Development Project of Shandong Province (2019GNC106089), and Foundation of Modern Agricultural Technology System Innovation Team Expert in Shandong Province (SDAIT-16-10).

\section{References}

[1] J. Lü, Z. Tian, and Y. Yang, "The development situation, existing problems and development trend of potato machinery," Journal of Agricultural Mechanization Research, vol. 12, pp. 258-263, 2015.

[2] X. Lu, "Strategy of potato as staple food: significance, bottlenecks and policy suggestions," Journal of Huazhong Agricultural University, vol. 3, pp. 1-7, 2015.

[3] Q. Duan and G. Sheng, "Research and design of 2CM-1 type potato planting machine," Journal of Chinese Agricultural Mechanization, vol. 37, no. 2, pp. 39-42, 2016.

[4] L. Shi, J. Wu, and W. Sun, "Simulation test for metering process of horizontal disc precision metering device based on discrete element method," Transactions of the Chinese Society of Agricultural Engineering, vol. 30, no. 8, pp. 40-48, 2014.

[5] J. Zhao, Y. Zhang, and L. Shen, "Design of the 2CMF-2 type potato planter," Modernizing Agriculture, vol. 4, pp. 37-38, 2009.

[6] W. Sun, G. Wang, and J. Wu, "Design and experiment on loss sowing testing and compensation system of spoon-chain potato metering device," Transactions of the Chinese Society for Agricultural Machinery, vol. 32, no. 11, pp. 8-15, 2016.

[7] M. V. Efimova, V. A. Khripach, E. V. Boyko et al., "The priming of potato plants induced by brassinosteroids reduces oxidative stress and increases salt tolerance," Doklady Biological Sciences, vol. 478, no. 1, pp. 33-36, 2018.

[8] C. Liu, S. Ravnskov, and F. Liu, “Arbuscular mycorrhizal fungi alleviate abiotic stresses in potato plants caused by low phosphorus and deficit irrigation/partial root-zone drying," The Journal of Agricultural Science, vol. 156, no. 1, p. 13, 2018.

[9] M. Zhang, X. Luo, and Z. Wang, "Design and experiment of combined hole-type metering device of rice hill-drop drilling machine," Transactions of the Chinese Society for Agricultural Machinery, vol. 47, no. 9, pp. 29-36, 2016.

[10] Q. Lai, W. Ma, and W. Su, "Design and experiment of pneumatic disc seed-metering device for mini-tuber," Transactions of the Chinese Society for Agricultural Machinery, vol. 47 , no. 12 , pp. 30-37, 2016.

[11] Z. Li, X. Wen, and J. Lü, "Analysis and prospect of research progress on key Technology and equipment of potato planting mechanization," Transactions of the Chinese Society of Agricultural Machinery, vol. 150, no. 3, pp. 1-16, 2019.

[12] J. Liu, T. Cui, and D. Zhang, "Mechanical-pneumatic combined corn precision seed-metering device," Transactions of the Chinese Society for Agricultural Machinery, vol. 43, no. 2, pp. 43-47, 2012, in Chinese.

[13] J. Hou, W. Liu, and W. Zhang, "Design of precision pneumatic cup seed-metering device for potato," Transactions of the Chinese Society of Agricultural Engineering, vol. 34, no. 24, pp. 18-28, 2018.

[14] J. Lü, Y. Yang, and Z. Li, "Design and experiment of cup-belt type potato seed-metering device," Transactions of the Chinese Society of Agricultural Engineering, vol. 32, no. 16, pp. 17-25, 2018.

[15] X. Wang, H. Tang, and J. Wang, "Optimized design and experiment on double-row cross spoon-belt potato precision seed metering device," Transactions of the Chinese Society for Agricultural Machinery, vol. 47, no. 11, pp. 82-90, 2018.

[16] W. Liu, J. He, and H. Li, "Design and experiment of vibrationarranging based seeder for potato micro-seed," Transactions of the Chinese Society for Agricultural Machinery, vol. 50, no. 8, pp. 70-80, 2019.

[17] Q. Lai, W. Ma, and Su. Liu, "Simulation and experiment on seed-filling performance of pneumatic disc seed-metering device for mini-tuber," Transactions of the Chinese Society of Agricultural Machinery, vol. 48, no. 5, pp. 44-53, 2017.

[18] J. Lü, S. Yi, and G. Tao, "Design and experiment of precision air-suction type planter for potato," Transactions of the Chinese Society of Agricultural Engineering, vol. 34, no. 10, pp. 16-24, 2018.

[19] Y. Zhou, "Research progress of domestic potato planting machinery," Agricultural Engineering, vol. 7, no. 2, pp. 29-30, 2017.

[20] Z. Li, Y. Li, and H. Meng, "Discussion on the status quo of potato sowing machinery research," Agricultural Machinery, vol. 8, pp. 109-110, 2016.

[21] S. Ma, "Research and analysis of mechanized seeding and seeding Technology of potato in China," Agricultural Development \& Equipment, vol. 12, p. 208, 2018.

[22] F. Dai and S. Xin, "Design and experiment of combined membrane covering potato seeding machine," Transactions of the Chinese Society of Agricultural Machinery, vol. 48, no. 3, pp. 76-83, 2017.

[23] J. Lü, Y. Yang, and Z. Li, "Design and experiment of seeding device for scoop potato seeder," Transactions of the Chinese 
Society of Agricultural Engineering, vol. 32, no. 16, pp. 17-25, 2016.

[24] X. Chen and L. Zhong, "Design and experiment of belt type seeding device for air suction type seed metering device," Transactions of the Chinese Society of Agricultural Engineering, vol. 28, no. 22, pp. 8-15, 2012.

[25] W. Liu, M. Zhao, and W. Wang, "Theoretical analysis and experiment of seeding performance of air suction type seeding device," Transactions of the Chinese Society of Agricultural Engineering, vol. 26, no. 9, pp. 133-138, 2010.

[26] J. Lü, P. Wang, and L. Yang, "Design and experiment of seed dressing device for scoop potato seeder," Transactions of the Chinese Society of Agricultural Machinery, vol. 50, no. 7, pp. 51-60, 2019.

[27] K. Niu, L. Zhou, and Y. Yuan, "Design and experiment of selfreplanting system of spoon-chain potato seed metering device," Transactions of the Chinese Society of Agricultural Machinery, vol. 47, no. S1, pp. 76-83, 2016.

[28] H. Jia, Y. Chen, and J. Zhao, "Design and experiment of gasabsorbing mechanical compound soybean precision seed metering device," Transactions of the Chinese Society of Agricultural Machinery, vol. 49, no. 4, pp. 75-86, 2018.

[29] Y. Wang, Y. Jin, and S. Luo, "Design and experiment of centralized precision soybean seed-metering device," Transactions of the Chinese Society for Agricultural Machinery, vol. 49, no. 6, pp. 112-118, 2018. 